\title{
Verificación de datos de tala ilegal mediante herramientas geoespaciales
}

\section{Use of geospatial tools to verify official illegal logging data}

\section{BYRON EFRÉN ROSERO MINDA ${ }^{* * a)}$, MARÍA DE DECKER(b)}

(a) Perito Topógrafo adscrito al Concejo de la Judicatura de Ecuador, Quito, Ecuador

(b) Universidad Estatal Amazónica (UEA), Calle Teniente Hugo Ortíz E45, Puyo-Ecuador

*geoasistencialegal@gmail.com

\section{RESUMEN}

Las tecnologías geoespaciales son ampliamente utilizadas en procesos gubernamentales de gestión territorial. Su creciente disponibilidad en los países del hemisferio sur amerita la formación de recursos humanos calificados para evitar riesgos en las acciones institucionales de control por errores $u$ omisiones que determinen litigios con los regulados. Como ejemplo, fue analizado el proceso sancionatorio 027-2015 de la Autoridad Ambiental Nacional (AAN) de Ecuador, que identificó aumento de la frontera agrícola por tala no autorizada en el Área de Amortiguamiento de la Reserva Ecológica El Ángel. Se planteó verificar la referencia espacial de los datos de ubicación obtenidos por la AAN y la delimitación del área de afectación ambiental, con la hipótesis de sobredimensionamiento. Con el uso de herramientas CAD, GIS, Google Earth y técnicas como fotointerpretación, georeferenciación y replanteo de coordenadas in situ, se llegó a determinar que el área de la afectación señalada por la AAN en 0,68 hectáreas (ha) fue sobredimensionada en 0,3785 ha $(55,04 \%)$ mientras que 0,3092 ha $(44,96 \%)$ si fueron deforestadas Se asume que las coordenadas obtenidas por la AAN no muestran el área real de afectación debido a probables errores humanos, instrumentales o ambientales en la toma datos. Las tecnologías geoespaciales se 
han convertido en cruciales en todos los niveles de gobierno y su uso requiere conocimientos especializados que favorecerán con menos errores y mayor efectividad en las tareas de regulación y control efectuadas por las instituciones gubernamentales.

Palabras clave: Tecnologías geoespaciales, tala ilegal, áreas protegidas, gobernanza

\section{ABSTRACT}

Geospatial technologies are widely used in government territorial management processes. Its increasing availability in the countries of the southern hemisphere, merits the training of qualified human resources to avoid risks in institutional control actions due to errors or omissions that determine litigation with the regulated parties. As an example, the sanctioning process 027-2015 of the National Environmental Authority (AAN) of Ecuador was analyzed, which identified an increase in the agricultural frontier due to unauthorized logging in the Buffer Area of the El Ángel Ecological Reserve. It was proposed to verify the spatial reference of the location data obtained by the AAN and the delimitation of the area of environmental impact, with the hypothesis of oversizing. With the use of CAD, GIS, Google Earth tools and techniques such as photointerpretation, georeferencing and staking of coordinates in situ, it was determined that the affected area indicated by the AAN at 0.68 ha was oversized by 0.3785 ha $(55.04 \%)$ while 0.3092 ha $(44.96 \%)$ were deforested. It is assumed that the coordinates obtained by the AAN do not show the real affected area due to probable human, instrumental or environmental errors in data collection. Geospatial technologies have become crucial at all levels of government and their use requires specialized knowledge that will lead to fewer errors and greater effectiveness in the regulation and control tasks carried out by government institutions.

Keywords: Geospatial technologies, illegal logging, protected areas, governance

\section{INTRODUCCIÓN}

Actualmente, se denominan ciencias de la información geográfica (Malczewski \& Rinner, 2015) o tecnologías geoespaciales (Muñiz et al., 2015) a aquellas que originan datos e información geoespacial que son ampliamente utilizados en procesos gubernamentales de gestión territorial. Sus aplicaciones van desde la administración de servicios de salud, emergencias, la protección ambiental (Obermeyer et al., 2016), planificación del uso del suelo (Dawwas, 2014) e incluso el esclarecimiento de hechos y búsqueda de evidencias para temas judiciales, recibiendo para este creciente campo de aplicación la denominación GIS forense (Elmes et al., 2014).

A pesar de la creciente disponibilidad de cada vez mejores herramientas geoespaciales para la toma de datos de superficie, tales como sistemas de información geográfica (GIS), sistemas de posicionamiento global (GPS), globos virtuales, drones, entre otros especialmente en los países del hemisferio sur, la disponibilidad de recursos humanos calificados aún no es suficiente (Flores et al., 2013; Obermeyer et al., 2016; Pánek, 2015). Esto supone un riesgo para la institucionalidad, pues las acciones de regulación y control establecidas desde los niveles gubernamentales serían 
insubsistentes (Dawwas, 2014) llegando incluso a establecerse litigios por errores u omisiones administrativos, en cuyo caso, y de forma contraproducente, esos mismos datos e información geoespacial correctamente fundamentados tendrán gran capacidad persuasiva y convincente en cortes y tribunales (Roedl et al., 2014)

Como muestra se analizó la resolución emitida en el año 2016 por la Autoridad Ambiental Nacional (AAN) referente al proceso administrativo 027-2015, donde se determinó sanciones por el aumento de la frontera agrícola por tala de bosques no autorizada, Este hecho sucedió dentro del Área de Amortiguamiento de la Reserva Ecológica El Ángel (REEA) ubicada en la provincia del Carchi, región Norte de Ecuador, y debido a los atributos naturales del área de amortiguamiento de la reserva (Ministerio del Ambiente, 2015), la AAN determinó restricciones sobre el uso del suelo, contrastando aquello con las prácticas agropecuarias de los pobladores locales.

Las evidencias fotográficas del incidente y coordenadas de ubicación obtenidas por funcionarios de la AAN fueron los argumentos que justificaron la sentencia y sanción administrativa. Por su parte, los regulados iniciaron en el año 2018 un nuevo proceso judicial ante el Tribunal Distrital de lo Contencioso Administrativo en el Distrito Metropolitano de Quito, donde refieren presuntas vulneraciones de hecho y de derecho por parte del Estado a través de la AAN, esgrimiendo como una de las pruebas la experticia pericial topográfica con el fin de verificar si los datos citados en el proceso de sanción fueron correctos.

Puesto que a la fecha de elaboración de este artículo este caso no ha sido resuelto, el alcance del análisis realizado plantea la hipótesis de un sobredimensionamiento del área de afectación que menciona el proceso 027-2015, determinándose como objetivos de este trabajo: a) analizar la referencia espacial de los datos de ubicación obtenidos por la $A A N$; b) determinar si el área de afectación ambiental fue correctamente delimitada

\section{METODOLOGÍA}

\section{Localización geográfica}

Administrativamente, el litigio ocurrió en la parroquia urbana 27 de septiembre de la ciudad de El Ángel, cantón Espejo, provincia del Carchi, región norte de Ecuador (Figura 1). El sector es conocido como "Chabayán Alto", ex Hacienda San Antonio de Chabayán. Geográficamente, se encuentra en un rango de altitud que va de los 3360 a 3400 m.s.n.m., ocupando una cresta divisoria de aguas de pendiente montañosa (37 \% a $58 \%$ ) la cual distribuye la escorrentía a dos quebradillas sin nombre, afluentes del río Bobo que nace en los páramos de la REEA. El sector posee un clima ecuatorial mesotérmico semi húmedo con rangos de precipitación de entre 750 a 1000 milímetros de lluvia promedio anual y temperaturas promedio de 9 a 10 grados centígrados (Ministerio del Ambiente, 2015). El Plan de Manejo de la REEA cita las especies vegetales representativas a Buddleja incana, B. pichinchensis, Columellia oblonga, Escallonia myrtilloides, Geranium ayavacense, Gynoxys acostae, G. cuicochensis, G. hallii, Hesperomeles ferruginea, H. obtusifolia, Luzula gigantea, Polylepis incana, P. lanuginosa, P. microphylla, P. pauta, P. reticulata, P. sericea, $P$. weberbaveri, Rubus coriaceus, Weinmannia fagaroides (Ministerio del Ambiente, 2015). 
Figura 1

Ubicación del área de afectación

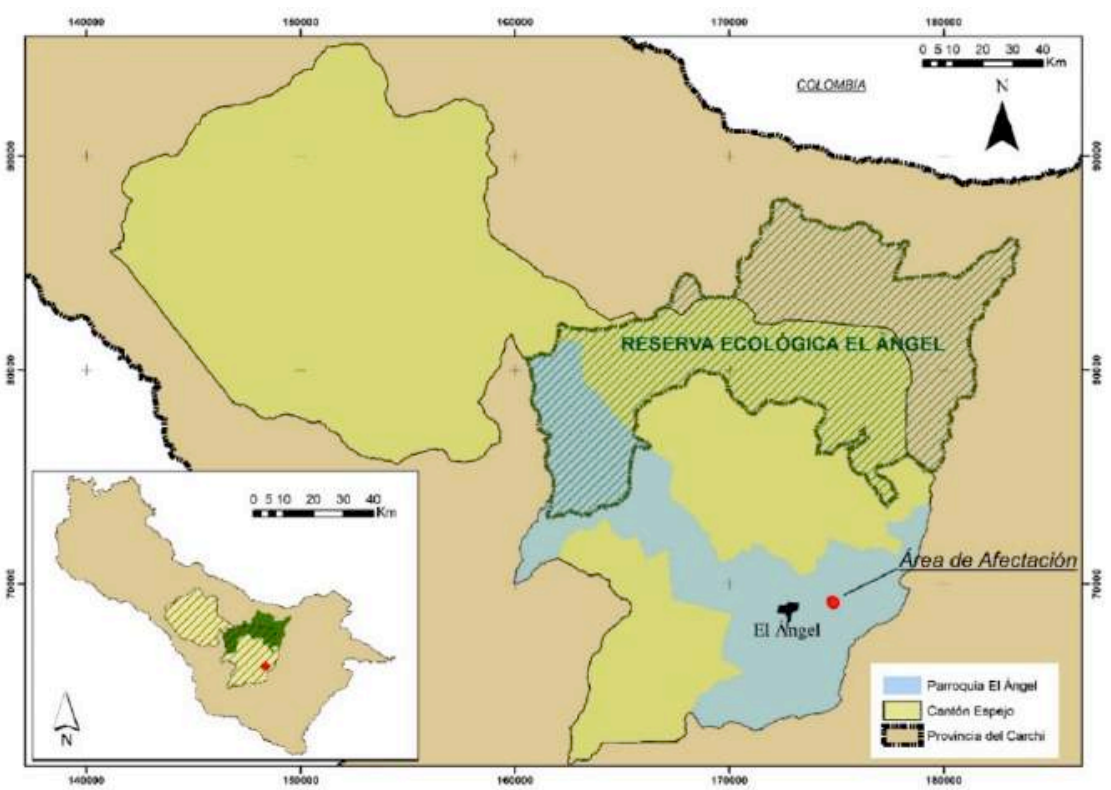

Nota. Reserva Ecológica El Ángel, ubicada en la provincia del Carchi, región Norte de Ecuador.

\section{Despliegue de coordenadas}

El proceso sancionatorio 027-2015 cita el informe técnico de Inspección por Tala llegal 1459-2015 donde se evidencia el aumento de la frontera agrícola realizado por los regulados en un área de 0,68 ha. Los funcionarios de la AAN obtuvieron las coordenadas de ubicación del incidente mediante receptor satelital tipo navegador y sus coordenadas se mencionan textualmente en la Tabla 1. Los receptores satelitales de baja precisión vulgarmente denominados "GPS" son frecuentemente útiles para actividades de manejo forestal relacionadas con la localización o mapeo de límites, topografía y levantamientos forestales catastrales (Yoshimura et al., 2002), inventario forestal, recursos y áreas de manejo especial (Wing et al., 2004) así como estimaciones de área y perímetro de bosque (Tachiki et al., 2005), entre otros.

Tabla 1

Coordenadas UTM del proceso 027-2015

\begin{tabular}{ccc}
\hline PUNTO & $\mathrm{X}$ & $\mathrm{Y}$ \\
\hline 1 & 842752 & 10069109 \\
2 & 842790 & 10069101 \\
3 & 842801 & 10069111 \\
4 & 842834 & 10069117 \\
5 & 842838 & 10069130 \\
6 & 842750 & 10069176 \\
7 & 842718 & 10069221 \\
8 & 842700 & 10069223 \\
9 & 842693 & 10069213 \\
10 & 842710 & 10069155 \\
11 & 842727 & 10069151 \\
12 & 842746 & 10069136 \\
13 & 842748 & 10069117 \\
\hline
\end{tabular}

Nota. Autoridad Ambiental Nacional de Ecuador (AAN) 
En el software AutoCAD se trazaron las coordenadas mencionadas y con polilínea se unieron estos vértices conformando un polígono de igual superficie al citado por la AAN. Debido a que el informe y proceso administrativos donde constan las coordenadas (Tabla 1) no mencionan íntegramente la referencia espacial del origen de los datos, mediante software ArcGIS se procedió a obtener la referencia espacial completa. Para ello, se descargaron datos cartográficos oficiales desde la página web del Instituto Geográfico Militar (IGM) con referencias espaciales conocidas para ser visualizados en ArcGIS conjuntamente con el polígono CAD ya trazado y las coordenadas UTM citadas por la AAN. Para el caso de las coordenadas el procedimiento seguido fue ingresarlas en una tabla de Excel (formato Libro de texto 1997-2003) y posteriormente desplegadas en el software ArcGIS con la función display XY Data y con referencia espacial extrapolada desde la cartografía oficial (UTM WGS84 Zona 17S). Luego, con un archivo shp tipo polígono se unieron estos vértices conformando nuevamente un polígono de igual superficie al citado por la AAN.

Para posibilitar el análisis del área de afectación sobre imágenes satelitales de Google Earth (GE), los archivos .shp de puntos y polígono ya georefenciados fueron re proyectados al sistema de coordenadas UTM de Google Earth (GE) que para la zona en estudio es UTM WGS84 zona 18 Norte. Posteriormente los archivos shp fueron transformados hacia el archivo nativo de GE denominado kml (por sus siglas en inglés: Keyhole Markup Language) El uso de estos archivos ya no se limita solo a las aplicaciones de Google Earth, porque varios sistemas de información geográfica (Global Mapper, AutoCAD Map, ArcGIS, etc.) pueden importar y exportar archivos KML (Balla \& Zichar,
2014). La conversión de un archivo .shp a un kml se realizó con la herramienta Layer to kml del módulo Conversion Tools en ArcToolbox para así visualizar el polígono de afectación en GE.

\section{Verificación de los datos}

Para verificar la geometría y localización de los datos $\mathrm{kml}$, fueron desplegados en GE por su agilidad para lograr observaciones interactivas con modelos digitales de terreno e imágenes de satélite de colores verdaderos (Selkin, 2016). Incluso con resoluciones bajas las imágenes de GE permiten ver fácilmente características importantes tanto del presente como del pasado reciente (Ballagh et al., 2011 ).

La imagen satelital empleada en GE fue a color, de alta resolución con GSD menor a $60 \mathrm{~cm}$ obtenida el 20 de noviembre de 2016 mediante sensores remotos de CNES-AIRBUS. Esta imagen con características de detalle a nivel parcelario y con una diferencia de 1 año y 22 días entre la fecha de la afectación y la fecha de toma, permitió realizar su análisis mediante técnica de fotointerpretación (Basterra, 2011).

Puesto que la confiabilidad de datos posicionales extraídos de GE u otras imágenes satelitales debe ser complementada con trabajo de campo para confirmar la veracidad de la información (Mohammed et al., 2013; Serrato, 2018), las coordenadas del lugar de los hechos fueron ingresadas a un receptor satelital tipo navegador marca Garmin, modelo Monterra con precisión +/- 2 m similar a los empleados por la AAN y a un equipo topográfico estación total marca Stonex, modelo R2 Plus con $2 \mathrm{~mm}$ de precisión de distancias. El receptor satelital sirvió 
además para definir los puntos de estacionamiento y orientación de este equipo. Con dicho instrumental, se aplicó la técnica de replanteo (Hurtares et al., 2011), tarea que fue realizada en el mismo lugar de los hechos. Estos equipos permitieron a distinto nivel de precisión verificar in situ parte de las coordenadas de la resolución, además de obtenerse coordenadas adicionales de los límites de la vegetación nativa remanente, del área despejada, el camino de ingreso y de las áreas cultivadas para verificar alineamiento y orientación de los datos proyectados a GE respecto a la imagen satelital, y así verificar e interpretar los cambios en la cobertura del suelo entre las fechas de ocurrencia de la afectación (octubre 2015), de toma de imagen satelital (noviembre 2016) y de realización de la experticia judicial (noviembre 2017).

\section{RESULTADOS}

\section{Reconstrucción del área de afectación}

El despliegue de coordenadas en AutoCAD permitió el trazado de un polígono de igual área a la que delimitó como afectación la AAN (Figura 2). Los archivos CAD de extensión .dwg fueron los datos espaciales básicos (Zhen et al., 2012) de partida para el análisis pericial.

\section{Identificación y definición de referencia espacial}

Losdatosquese proyectencorrectamentegarantizan representaciones y mediciones precisas (ESRI, 2019) y su correcta ubicación en un
Figura 2

Trazado de un polígono de igual área a la que delimitó como afectación la AAN

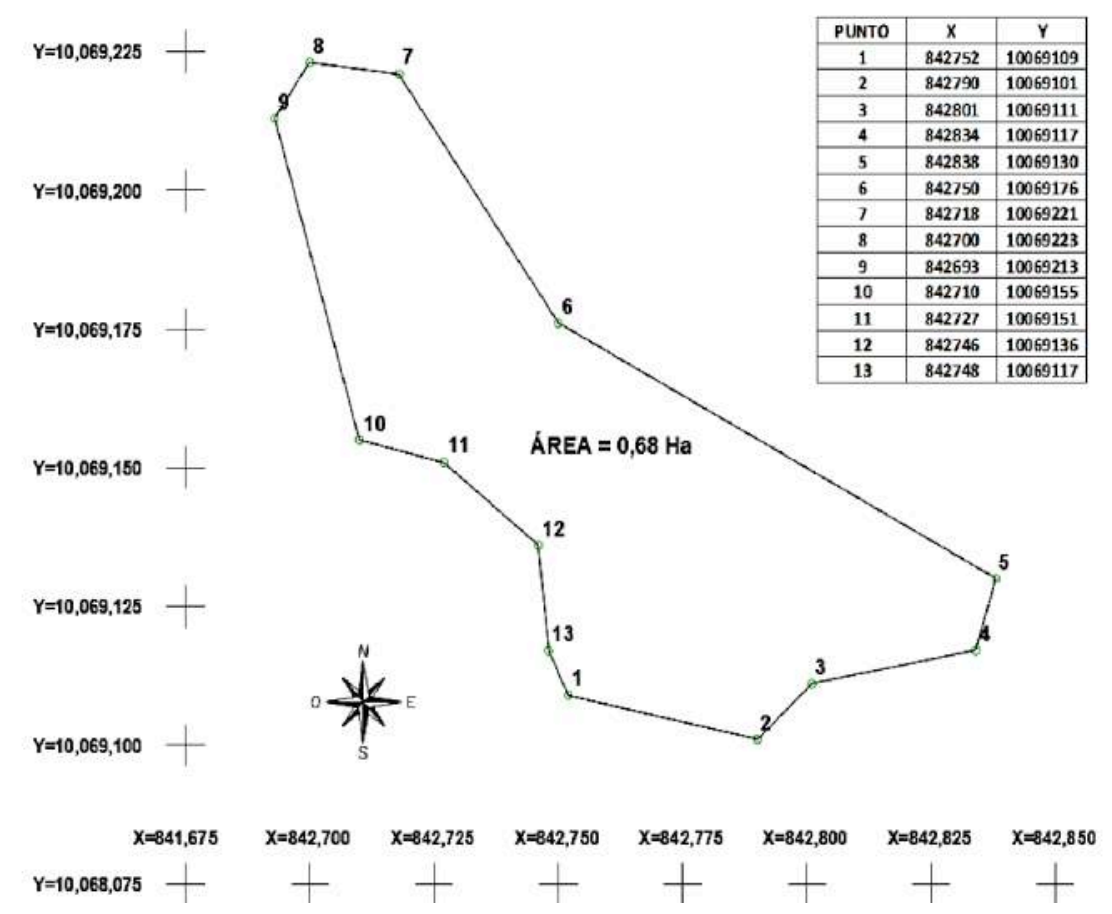

Nota. Trazado CAD del polígono de 0,68 ha con base en las coordenadas de la resolución 027-2015 de la AAN.

GIS permitirá correlacionarlos, fusionarlos y gestionarlos para explorar relaciones entre ellos (Elmes et al., 2014). Puesto que las coordenadas citadas en el proceso 027-2015 carecen de una completa referencia espacial, al visualizarse conjuntamente en GIS como archivos .dwg y .shp conservaron las características geométricas, pero sin definirse sistema de coordenadas (Figura 3). 
Figura 3

Características geométrico espaciales de los archivos .dwg y .shp del área de afectación

$$
\text { Extent }
$$

Top: $10069223,000000 ? ?$

Left: $842693,000000 ? ?$ Right: $842838,000000 ? ?$

Bottom: 10069101,000000 ??

Data Source
\begin{tabular}{|ll}
\hline Data Type: & CAD Polygon Feature Class \\
Location: & F:\} $\\
{\text { Feature Dataset: }} &{\text { ÁREA DE AFECTACIÓN. Jwa }} \\
{\text { Feature Class: }} &{\text { Polygon }} \\
{\text { Feature Type: }} &{\text { Simple }} \\
{\text { Geometry Type: }} &{\text { Polygon }} \\
{\text { Coordinates have Z values: }} &{\text { No }} \\
{\text { Coordinates have measures: }} &{\text { No }} \\
{\text { No Default Projection File }} &{ } \\
{\hline}$
\end{tabular}

Extent Top: $10069223,000000 ? ?$

Left: 842693,000000 ?? Right: $842838,000000 ? ?$

Bottom: 10069101,000000 ??

Data Source
\begin{tabular}{|ll|}
\hline Data Type: & Shapefile Feature Class \\
Shapefile: & F:AREA_DE_AFECTACION. hhp \\
Geometry Type: & Polygon \\
Coordinates have Z values: & No \\
Coordinates have measures: & No \\
& \\
Coordinate System: & <Undefined >
\end{tabular}

Nota. Se aprecia que el sistema de coordenadas no ha sido definido en los dos casos indicando interrogantes sobre su verdadera ubicación.
Figura 4

Características geométrico espaciales del archivo .shp del área de afectación
Extent

Left: $842693,000000 \mathrm{~m}$

Top: $10069223,000000 \mathrm{~m}$

Bottom: $10069101,000000 \mathrm{~m}$

Data Source
\begin{tabular}{|ll}
\hline & \\
\hline Projected Coordinate System: & WGS_1984_UTM_Zone_17S \\
Projection: & Transverse_Mercator \\
False_Easting: & 500000,00000000 \\
False_Northing: & 10000000,00000000 \\
Central_Meridian: & $-81,000000000$ \\
Scale_Factor: & 0,99960000 \\
Latitude_Of_Origin: & 0,00000000 \\
Linear Unit: & Meter
\end{tabular}

Nota. Se aprecia que el sistema de coordenadas ha sido correctamente definido con la Proyección UTM, Datum WGS84 y Zona 17 Sur, indicándose la extensión real del área de afectación.

\section{Transformación de referencia espacial y análisis en GE}

Sin una apropiada combinación de proyección, datum y sistema de coordenadas, no se tiene la habilidad de sobreponer datos geográficos (Dixon \& Uddameri, 2016) y analizarlos correctamente con otros datos. Definidas las referencias espaciales del área afectación y re proyectadas como UTM Datum WGS84 Zona 17 Sur, al transformarlas al sistema UTM de Google Earth se obtuvo para fines de comparación las coordenadas en los dos sistemas de referencia espacial citadas en la Tabla 2: 
Tabla 2

Coordenadas XY con dos sistemas de referencia espacial UTM

\begin{tabular}{ccccc}
\hline & \multicolumn{2}{c}{ Datum WGS84 Zona 17 Sur } & \multicolumn{2}{c}{ Datum WGS84 Zona 18 Norte } \\
\hline PUNTO & $\mathrm{X}$ & $\mathrm{Y}$ & $\mathrm{X}$ & $\mathrm{Y}$ \\
\hline 1 & 842752 & 10069109 & 174834 & 69099 \\
2 & 842790 & 10069101 & 174872 & 69091 \\
3 & 842801 & 10069111 & 174883 & 69101 \\
4 & 842834 & 10069117 & 174916 & 69107 \\
5 & 842838 & 10069130 & 174920 & 69120 \\
6 & 842750 & 10069176 & 174832 & 69166 \\
7 & 842718 & 10069221 & 174800 & 69211 \\
8 & 842700 & 10069223 & 174782 & 69213 \\
9 & 842693 & 10069213 & 174775 & 69203 \\
10 & 842710 & 10069155 & 174792 & 69145 \\
11 & 842727 & 10069151 & 174809 & 69141 \\
12 & 842746 & 10069136 & 174828 & 69126 \\
13 & 842748 & 10069117 & 174830 & 69107 \\
\hline
\end{tabular}

Nota. Referencias espaciales del área de afectación y re proyectadas como UTM Datum WGS84 Zona 17 Sur, al transformarlas al sistema UTM de Google Earth se obtuvo para fines de comparación las coordenadas en los dos sistemas de referencia espacial.

Con la re proyección de los datos de coordenadas (y polígono de afectación) a la referencia espacial de GE, se logró sobreponerlos en una imagen satelital tomada el 20 de noviembre de 2016 (388 días después de la afectación) identificándose que el polígono de afectación además de incluir las áreas deforestadas, también englobó parte de parcelas de cultivos, el camino de tercer orden de ingreso al lugar y remanentes del bosque alto andino junto al camino (Figura 5).
Figura 5:

Coordenadas y polígono de afectación sobre imagen satelital en Google Earth

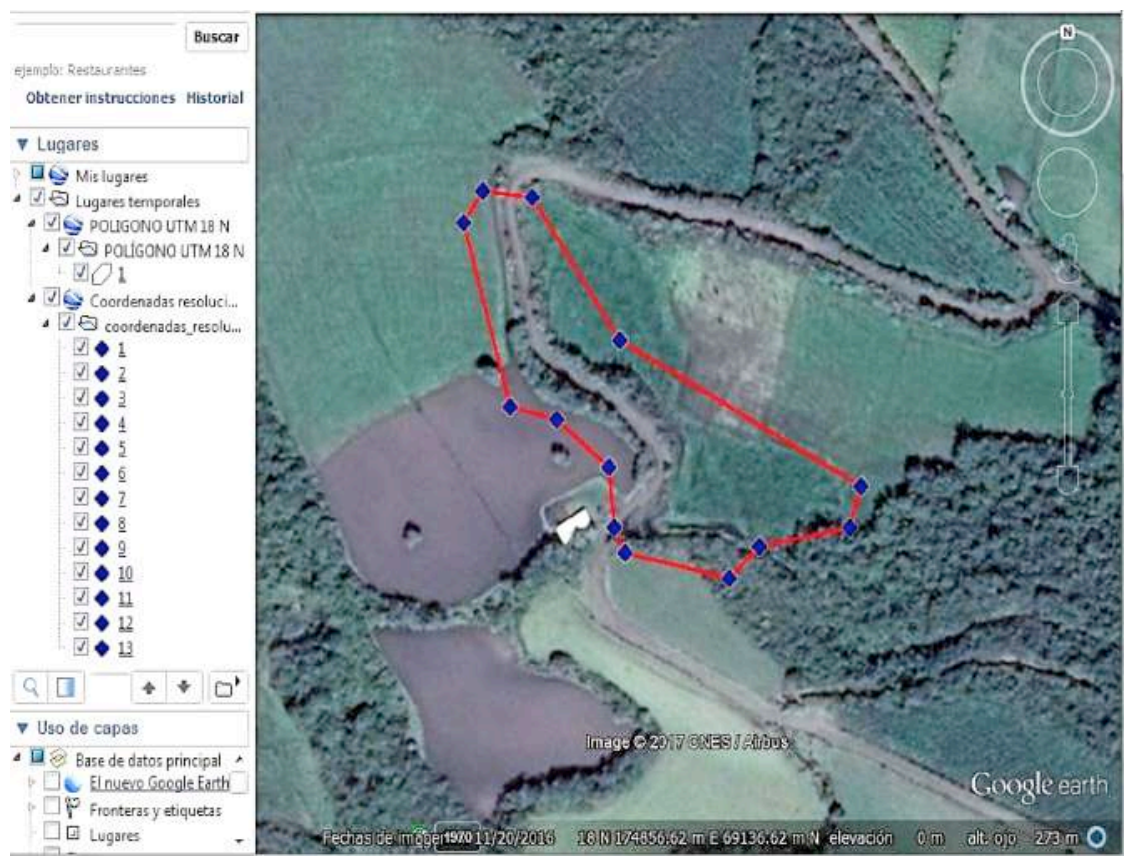

Nota. Polígono de afectación además de incluir áreas deforestadas, parcelas de cultivos, camino de tercer orden de ingreso al lugar y remanentes del bosque alto andino junto al camino

Como se puede observar en la figura 5, las coordenadas y por ende, el polígono de afectación no coinciden con los límites como el camino o línea del bosque, asumiendo que las coordenadas obtenidas por la AAN no muestran el área real de afectación debido probablemente a errores humanos, instrumentales o ambientales que afectaron la precisión del receptor satelital tipo navegador que debió ser utilizado. Las diferencias entre ubicaciones registradas y ubicaciones reales obtenidas con receptores tipo navegador con 
señal GPS muestran diferencias en el plano horizontal de entre 4,8 y 30,6 metros (Deon et al., 2002; Rodríguez Pérez et al., 2006). El mayor factor de error que afecta la exactitud y la precisión del posicionamiento es la falta de la cantidad de satélites (Yamaguchi \& Tanaka, 2006) y una débil señal (Thin et al., 2016).

También se asume que el error por defecto de los receptores satelitales empleados sumado a factores ambientales como la densa nubosidad registrada en octubre un mes lluvioso en esta zona ubicada sobre los 3000 m.s.n.m (Ministerio del Ambiente, 2015) o el efecto ionosférico que causa errores significativos de \pm 5 metros debido a la refracción de las ondas electromagnéticas de los satélites (Thin et al., 2016), pudieron haber contribuido al problema por acumulación de errores.

\section{Figura 6}

Areas y porcentajes de usos del suelo dentro del polígono citado como afectación por parte de la AAN

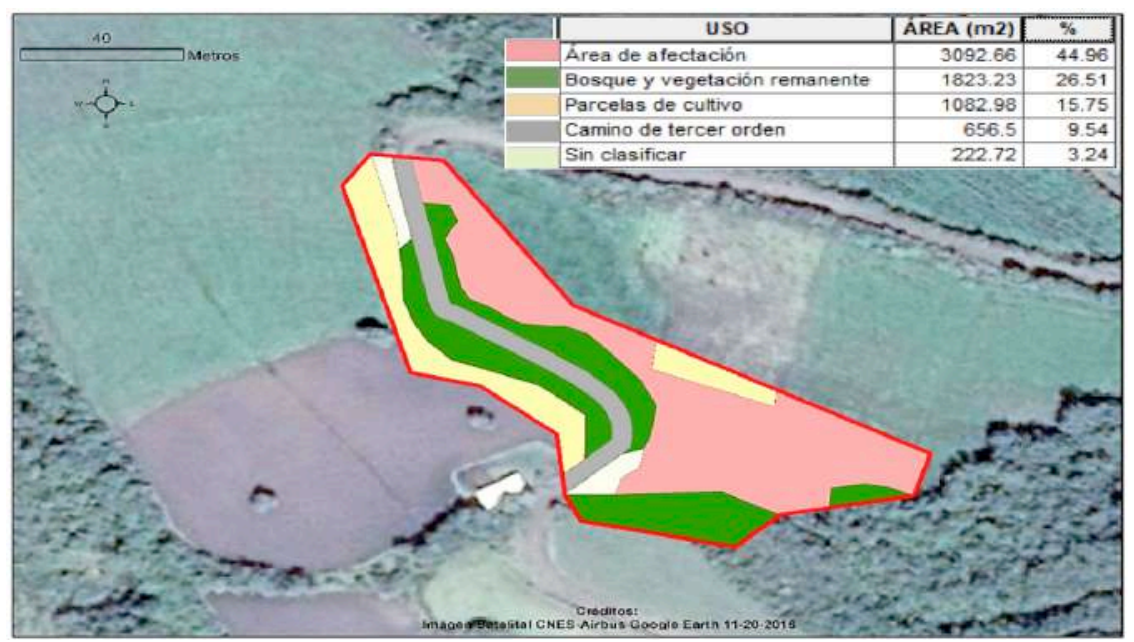

Nota. Porcentajes del área de afectación, bosques y vegetación con remanente, parcelas de cultivo, camino de tercer orden de ingreso al lugar y sin clasificar.

\section{Uso de suelo y área de afectación}

Mediante la fotointerpretación satelital y el cálculo de áreas obtenidas se determinó que el área de afectación por deforestación fuese 3092,66 m2, es decir, el 44,96 \% del área señalada por la AAN. El $55,04 \%$ de diferencia es decir 3785,43 m2 correspondió a bosque y vegetación remanente con el 26,51 \% (1823,23 m2), parcelas de cultivos con 15,75 \% (1082,98 m2), el camino de tercer orden a $9,54 \%(656,5 \mathrm{~m} 2)$ y uso sin clasificar con el 3,24 \% (222,72 m2).

El área obtenida por la AAN como afectación fue 0,68 ha mientras que el área de afectación obtenida pericialmente fue 0,32 ha existiendo una diferencia de 0,36 ha de exceso señalado como tala ilegal, comprobándose la hipótesis planteada en este trabajo.

Figura 7

Afectación según AAN

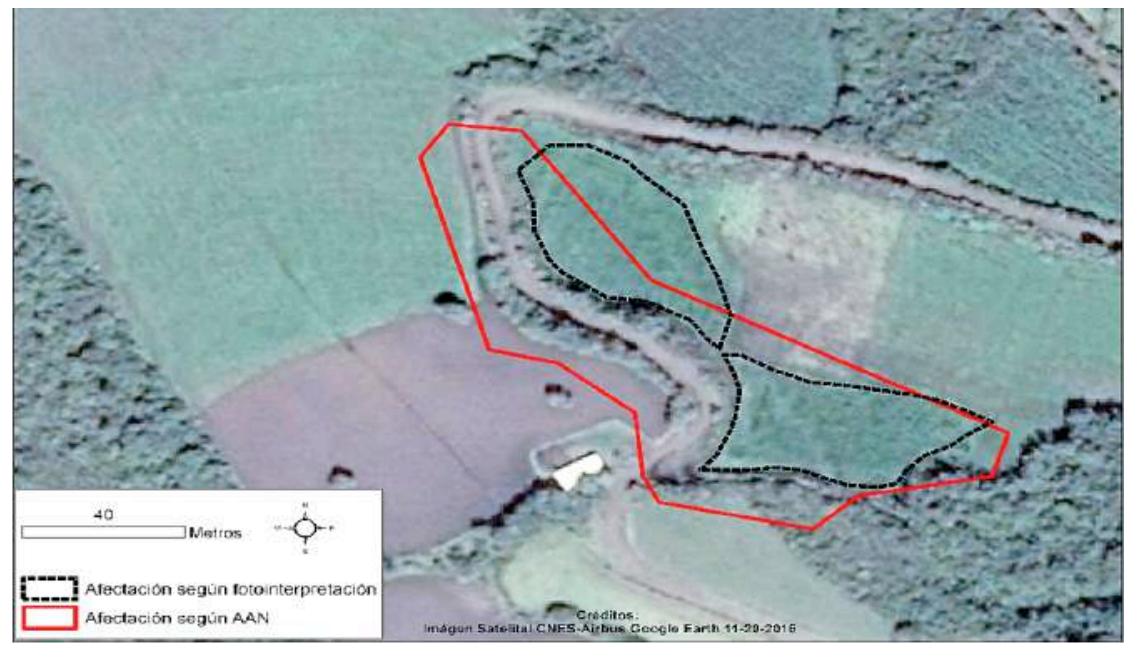

Nota. Afectación según AAN (polígono rojo) y afectación según experticia pericial (polígonos negros) obtenidos mediante técnica de fotointerpretación y verificada in situ 


\section{DISCUSIÓN}

Al emplear Sistemas de Información Geográfica, las entidades gráficas que representan los objetos del mundo real deben poseer una referencia espacial que describa el lugar de ubicación de dichas entidades (ESRI, 2016). Al hablar de referencia espacial se refiere a un sistema que involucra sistema de coordenadas, proyección y datum (Jiang \& Li, 2014). El proceso administrativo 027-2015 menciona 13 pares de coordenadas que demarcaron el área de afectación, pero sin mencionar la totalidad de la referencia espacial pues se menciona únicamente la proyección UTM. Puesto que un sistema de coordenadas es un sistema de referencia que se utiliza para representar la ubicación de las entidades geográficas, imágenes y ubicaciones de GPS en un marco geográfico común (ESRI, 2016), la omisión de este marco geográfico común dentro de las pruebas anunciadas en el ámbito legal podría constituir un argumento en contra. Si dicho aspecto fuese expuesto por un testigo experto de los tribunales o perito, el medio acusatorio en un alegato judicial podría ser negado o descartado por el juzgador (Rey Navas, 2017).

La fotointerpretación de imagen satelital de resolución a nivel de parcela permitió identificar áreas que no fueron taladas pero que fuesen incluidas como afectación por los funcionarios de la AAN. Se determinó que solo el 44,96 \% del área fue talada, mientras que el 55,04 \% correspondió a otros usos de suelo incluido el camino de acceso al lugar. Para corroborar estas conclusiones, in situ y mediante estación total y navegador, fueron replanteadas dos pares de coordenadas del polígono y adicionalmente se obtuvo coordenadas de la línea del bosque existente hasta noviembre de 2017 y parte del camino de acceso verificándose así la ubicación del bosque maduro y el alineamiento de la imagen satelital empleada respecto al polígono de afectación determinado por la AAN.

Figura 8

Datos de replanteo de vértices del polígono y levantamiento topográfico del camino.

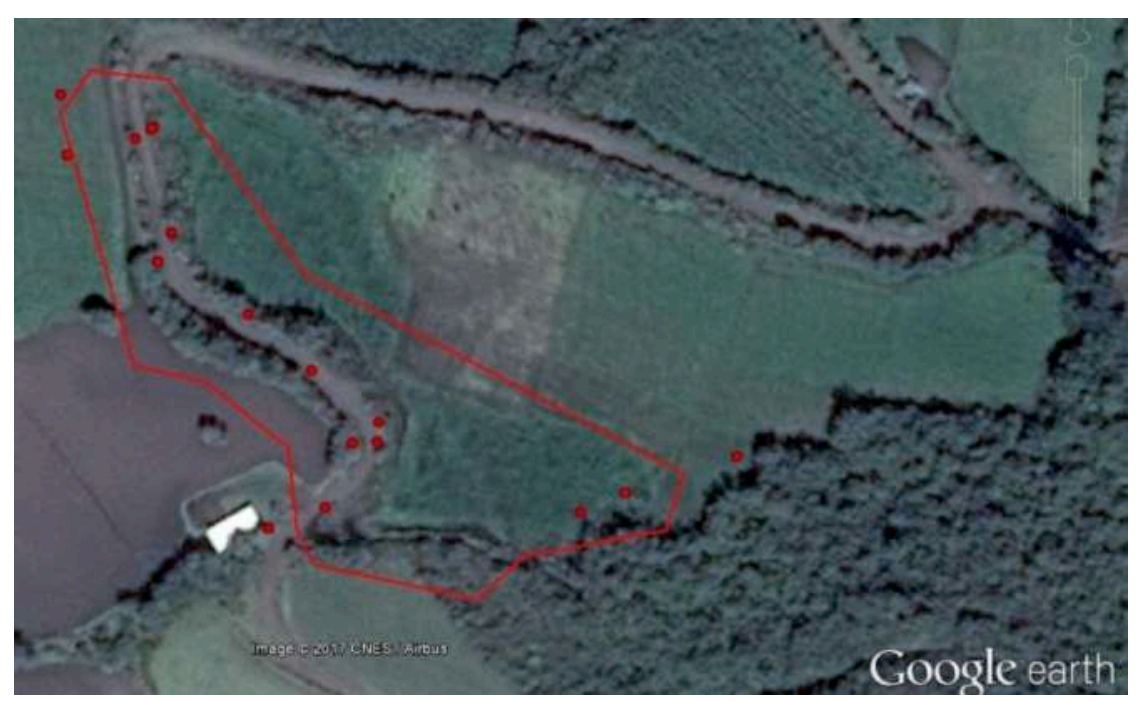

Nota. Datos de replanteo de vértices del polígono y levantamiento topográfico del camino, y línea de bosque existente hasta noviembre de 2017 adjuntos al polígono de afectación según la AAN.

Finalmente, de aceptar el área propuesta por la AAN implicaría que parte de las parcelas de cultivos y el camino de ingreso en su interior estuvieron cubiertas de vegetación nativa. A lo cual, se debe recordar que el tiempo trascurrido entre la fecha de 
afectación (octubre 2015) y la fecha de toma de la imagen satelital empleada en este trabajo fue solo 387 días, tiempo en el cual es imposible que la vegetación leñosa o arbustiva alto andinas crezca en tan poco tiempo (Castellanos-Castro \& Bonilla, 2011). Al tratarse de remanentes de vegetación resultado del desarrollo de la actividad agropecuaria en el sector, ocasionará que el proceso de regeneración natural se retrase aún más debido a las barreras ecológicas como la competencia, la disminución de dispersores o la fragmentación (Acosta Ortiz \& Vargas, 2008) que produce la actividad agropecuaria.

\section{CONCLUSIÓN}

Debido a que las ciencias y tecnologías geoespaciales se han convertido en cruciales en todos los niveles de gobierno, su uso requiere conocimientos especializados (Obermeyer et al., 2016).

Emplear sistemas de posicionamiento global tales como GPS, GLONASS, EGNOSS, GALILEO (Peñafiel \& Zagas, 2001) requiere considerar factores humanos, ambientales e instrumentales que pueden afectar la calidad de las señales satelitales (El-Rabbany, 2002). Sobre este último factor la calidad de los receptores incide significativamente pues no es lo mismo colectar datos con receptores tipo navegador a utilizar receptores de doble frecuencia (Schwieger, 2003) aunque en ambos casos se enfrentan desafíos para un uso efectivo en entornos boscosos (Wing et al., 2004). Por estas razones, el uso de un único dispositivo satelital de toma de coordenadas para obtener evidencias no es suficiente requiriéndose el empleo de otros instrumentos tales como estaciones totales, drones y comparación de imágenes satelitales.
La falta de programas de adiestramiento o capacitación en las instituciones públicas pondrán en riesgo la efectividad de las acciones de control, resultando los esfuerzos de regulación gubernamental insubsistentes.

\section{BIBLIOGRAFÍA}

Acosta Ortiz, M., \& Vargas, O. (2008). Ampliación de Fragmentos de Bosque Altoandino. In Estrategias para la restauración ecológica del bosque altoandino (Vol. 64, Issue 2, pp. 266-281). Universidad Nacional de Colombia. https://www.researchgate. net/publication/263658634_Ampliacion_de_Fragmentos_de Bosque_Altoandino

Balla, D., \& Zichar, M. (2014). Geovisualization of domestic nature conservation areas using KML. 9th International Conference on Applied Informatics Eger. Hungary, 185-193.

Ballagh, L. M., Raup, B. H., Duerr, R. E., Khalsa, S. J. S., Helm, C., Fowler, D., \& Gupte, A. (2011). Representing scientific data sets in KML. Computers \& Geosciences, 37(1), 57-64.

Basterra, I. (201 1). Cátedra de Fotinterpretación.

Castellanos-Castro, C., \& Bonilla, M. A. (2011). Grupos Funcionales de Plantas con Potencial Uso para la Restauración en Bordes de Avance de un Bosque Altoandino. Acta Biologica Colombiana, 16(1), 153-174.$$
6(1), 153-174 .
$$ 
Dawwas, E. (2014). The Evolution of GIS as a Land Use Planning Conflict Resolution Tool: A Chronological Approach. American Journal of Geographic Information System, 3(1), 38-44. https:// doi.org/10.5923/i.ajgis.20140301.04

Deon, R., Serrouya, R., \& Kochanny, C. (2002). GPS radiotelemetry error and bias in mountainous terrain. Wildlife Society Bulletin, 30(2), 430-439. https://doi.org/10.2307/3784501

Dixon, B., \& Uddameri, V. (2016). GIS and Geocomputation for Water Resource Science and Engineering. In GIS and Geocomputation for Water Resource Science and Engineering. https://doi. org/10.1002/9781118826171

El-Rabbany, A. (2002). Introduction to GPS. Artech House. http://www.ghbook.ir/index.php?name= مناسروكننرف نونىونىاه $\mathrm{id}=13650$ \&page $=73 \&$ chkhashk $=$ ED9C9491 B4\&ltemid $=$ $218 \&$ lang $=$ fa\&tmpl $=$ component

Elmes, G., Roedl, G., \& Conley, J. (2014). Forensic GIS (G. Elmes, G. Roedl, \& J. Conley (eds.)). Springer. https://doi. org/10.1007/978-94-017-8757-4

ESRI. (2016). ¿̇Qué son las proyecciones cartográficas —Ayuda ArcGIS for Desktop. http://desktop.arcgis.com/es/arcmap/10.3/guidebooks/map-projections/what-are-map-projections.htm

ESRI. (2019). Información general sobre el conjunto de herramientas Proyecciones-Ayuda Documentación. Información General Sobre El Conjunto de Herramientas Proyecciones. https:// desktop.arcgis.com/es/arcmap/latest/tools/coverage-toolbox/ define-projection.htm
Flores, F., Araque, F., Entrena, M., \& Aguilar, R. (2013). Capacitación en Geomática para el personal del sector público en Venezuela Artículos. GEOENSEÑANZA, 18, 81-89. http://webcache.googleusercontent.com/ search? $q=$ cache:AHZWoE 8 pxNAJ:www.saber.ula.ve/ bitstream/handle/123456789/42268/articulo_5c.pdf\% 3Fsequence\%3D2\%26isAllowed\%3Dy $+\& c d=1 \& \mathrm{hl}=$ es $419 \& c t=c \operatorname{lnk} \& g l=e c \& c l i e n t=$ firefox $-b-d$

Hurtares, W., Chilán, K., Solórzano, I., \& Santos, E. (2011). Trazado y Replanteo del Proyecto Horizontal de una carretera utilizando Estación Total. Research Gate, 1. https://www.researchgate.net/ publication/277985741_Trazado_y_replanteo_del_proyecto_ horizontal_de_una_carretera_utilizando_estacion_total_caso practico_camino_vecinal_la_chorera_km_0000_-_2187

Jiang, W., \& Li, J. (2014). The effects of spatial reference systems on the predictive accuracy of spatial interpolation methods. 33. https://pdfs.semanticscholar.org/6e70/ fe533372a4bcaf647533425cb85a839b17f1.pdf

Malczewski, J., \& Rinner, C. (2015). Multicriteria Decision Analysis in Geographic Information Science. In Springer (Ed.), Analysis methods (Issue Massam 1993). https://doi.org/10.1007/9783-540-74757-4

Ministerio del Ambiente. (2015). Plan de Manejo Reserva Ecológica El Ángel. In Latindex (Vol.1). http://suia.ambiente.gob.ec/ documents/10179/242256/24 + PLAN + DE + MANEJO + EL+ANGEL.pdf/134234ba-a47d-459c-956b-37b0155df002 
Mohammed, N. Z., Ghazi, A., \& Mustafa, H. E. (2013). Positional accuracy testing of Google Earth. International Journal of Multidisciplinary Sciences and Engineering, 4(6), 6-9. http:// www.ijmse.org/Volume4/Issue6/paper2.pdf

Muñiz, O., Demirci, A., \& Schee, J. van der. (2015). Geospatial Technologies and Geography Education in a Changing World. Geospatial Practices and Lessons Learned (O. Muñiz, A. Demirci, \& J. van der Schee (eds.)). https://doi.org/10.1007/978-4-431 55519-3

Obermeyer, N. J., Ramasubramanian, L., \& Warnecke, L. (2016). GIS Education in U. S. Public Administration Programs: Preparing the Next Generation of Public Servants. Journal of Public Affairs Education, 22(2), 249-266. https://doi.org/10.1080/152368 03.2016 .12002244

Pánek, J. (2015). Geographic Information Systems in Developing Countries - What are the Ethical Issues we need to be aware of ? / Geografické Informační Systémy A Jejich Využití V Rozvojových Zemích - Jakým Etickým Otázkám Musíme Čelit? GeoScience Engineering, 59(2), 40-48. https://doi.org/10.2478/gse2014-0052

Peñafiel, J., \& Zagas, J. (2001). Fundamentos del sistema gps y aplicaciones en la topografia. In Colegio Oficial de Ingenieros Tecnicos en Topografia.

Rey Navas, F. I. (2017). Pertinencia del testigo experto, testigo perito y testigo de refutación en la teoría del caso. Revista de Derecho Principia luris, 15. revistas.ustatunja.edu.co > index.php > piuris > article , download
Rodríguez Pérez, J., Álvarez Taboada, M., Sanz Ablanedo, E., \& Gavela, A. (2006). Comparison of GPS receiver accuracy and precision in forest environments. Practical recommendations regarding methods and receiver selection. XXIII International FIG Congres, 1-16. https://www.mendeley.com/reference-manager/reader/ d87eed0e-e659-3df8-al6f-c283bld7ac86/6a40d874-106eec4b-6fld-7e4c1 be22b9f

Roedl, G., Elmes, G. A., \& Conley, J. (2014). Geospatial Technologies in the Courtroom. In Forensic GIS (p. 314). Springer.

Schwieger, V. (2003). Using Handheld GPS Receivers for Precise Positioning. 2nd FIG Regional Conference, February, 1-16.

Selkin, P. (2016). Google Earth and Geoscience Education. Teach the Earth. http://serc.carleton.edu/NAGTWorkshops/visualize04/ tool_examples/google_earth.html

Serrato, P. (2018). Conceptos Basicos De Interpretacion De Aerofotografías E Imágenes SatelitaleS. https://doi.org/. DOI: 10.13140/RG.2.2.18697.08809

Tachiki, Y., Yoshimura, T., Hasegawa, H., Mita, T., Sakai, T., \& Nakamura, F. (2005). Effects of polyline simplification of dynamic GPS data under forest canopy on area and perimeter estimations. Journal of Forest Research, 10, 419-427.

Thin, L. N., Ting, L. Y., Husna, N. A., \& Husin, M. H. (2016). GPS systems literature: Inaccuracy factors and effective solutions. International Journal of Computer Networks and Communications, 8(2), 123-131. https://doi.org/10.5121/ijenc.2016.8211 
Wing, M. G., Solmie, D., \& Kellogg, L. (2004). Comparing Digital Range Finders for Forestry Applications. Journal of Forestry, 102(4), 16-20. https://doi.org/10.1093/jof/102.4.16

Yamaguchi, S., \& Tanaka, T. (2006). GPS standard positioning using Kalman filter. SICE-ICASE International Joint Conference, 13511354. https://doi.org/10.1109/SICE.2006.315572.

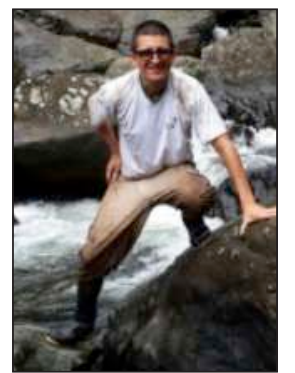

\section{BYRON EFRÉN ROSERO MINDA}

- Amante de la Naturaleza, ciudadano del mundo, hombre de familia, profesional y emprendedor.

- He desarrollado su trabajo en organismos públicos, privados, organizaciones comunitarias y la cooperación internacional durante 10 años, tanto en la Región Norte como en la Amazonía de Ecuador.

- Ejerció como docente en varias universidades en asignaturas como: Introducción a la Ingeniería Ambiental, Sistemas de Información Geográfica, Ordenamiento Territorial, Cartografía y Topografía y Dibujo Técnico. Actualmente es emprendedor en el ámbito privado prestando servicios profesionales como topógrafo y avaluador tareas que también las realiza en juzgados, fiscalías y tribunales en calidad de perito calificado del Consejo de la Judicatura.

Ingeniero en Recursos Naturales por la Universidad Técnica del Norte y Magister en Desarrollo Regional y Planificación Territorial por la Pontificia Universidad Católica del Ecuador.
Yoshimura, T., Gandaseca, S., Gumus, S., \& Acar, H. (2002). Evaluating the accuracy of GPS positioning in the forest of the Macka regio. The Second National Black Sea Forestry Congress Proceedings $1,62-69$.

Zhen, L., Jing, C., \& Chen, X. (2012). Files' conversion from CAD to GIS using spatial data conversion tools provided by FME. Proceedings - 2012 International Conference on Computer Science and Service System, CSSS 2012, 1939-1942. https:// doi.org/10.1109/CSSS.2012.484

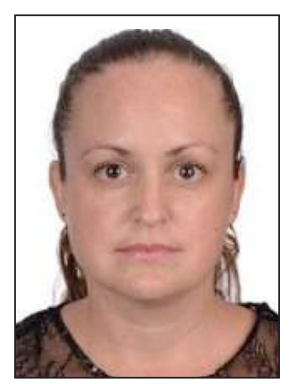

\section{MARÍA DE DECKER}

- Máster en Ciencias Ambientales, con especialización en Planificación del Desarrollo para Zonas Rurales. Tiene experiencia laboral como educadora en varias instituciones de educación superior como: Universidad Estatal Amazónica (UEA), desde septiembre 2015 con las asignaturas "Ordenamiento Territorial", "Cartografía y Topografía", en las carreras Ambiental y Agropecuaria; "Sistemas de Información Geográfica", en las carreras Ambiental y Biología, "Topografía Forestal y SIG" y "Fotogrametría forestal y Fotointerpretación", en la carrera Forestal. Ha participado en el proyecto de investigación LaForeT (Landscape Forestry in the Tropics) - 2016. 2018, coordinado por la UEA y el Instituto Von Thünen, Alemania. Investigadora de varios proyectos sobre el Cambio Climático (CEPLAES, COICA) y traductora alemán, español e inglés (por ejemplo, de artículos científicos) 